\title{
Eigenvalue Distribution Conditions for Some Classes of Interval Matrices
}

\author{
Takehiro MORI* and Hideki KOKAME**
}

\begin{abstract}
It is known that symmetric interval matrices are Hurwitz stable or positive definite if and only if all the vertex symmetric matrices are so. This paper generalizes this fact in two directions. Interval matrices dealt with here are such a class of matrices that have only real eigenvalues, thus including symmetric interval matrices. The other generalization is made by taking account of eigenvalue distribution in a more general manner. Namely, we consider the situation where, for some real $k$, a certain number of eigenvalues are located in the left of $k$ while the remaining ones in the right of $k$. An imaginary axis couterpart of the obtained result is also provided.
\end{abstract}

Key Words : interval matrices, eigenvalue distribution, real eigenvalues, aperiodicity

\section{Introduction}

A possible way to represent parametric uncertainties involved in systems is to use interval parameters. This assumes that we can somehow estimate upper and lower bounds for the parameters. In case when the parameters are coefficients of their charcacteristic polynomials, the representation gives rise to interval polynomials, for which the renowned Kharitonov theorem gives quite compact Hurwitz stability conditions in terms of stability of vertex polynomials ${ }^{5}$. However, for interval matrices, the state-space counterpart, we can expect no such nice parallels to polynomial case in general. For instance, Hurwitz stability of all the vertex matrices by no means implies that of interval matrices. This becomes, however, correct, if we restrict interval matrices to be symmetric.

The purpose of the present paper is to show that this fact can be more generalized. The generalization is made in two directions. One is a point that we can deal with not only symmetric interval matrices but also a class of interval matrices whose eigenvalues

\footnotetext{
* Dept. of Electronics \& Information Science, Kyoto Institute of Technology, Kyoto

** Dept. of Electrical Engineering, Osaka Institute of Technology, Osaka

(Received September 11, 1992)

(Revised December 7, 1992)
}

are all real. The other generalization is made by taking a more general eigenvalue configuration on the real line into account. We will consider the situation where, for a given real $k$, a certain number of eigenvalues are in the left hand side of $k$ while the others are in the right. This enables us, for example, to derive a continuous-time or discrete-time aperiodicity condition for interval state-space models. We furthermore show that the similar result holds for interval matrices that have all their eigenvalues on the imaginary axis. The construction of this paper is as follows. In the next section, we list up symbol conventions and formulate the present problem in association with some previous work. Section 3 contains main results including the imaginary axis counterpart. Several remarks on these results and a numerical example are povided in section 4 . We draw some conclusions in Section 5.

\section{Preliminaries}

Interval matrix is a set of real matrices whose entries are within specified intervals on the real line and is often expressed as $N[P, Q]$ using two most lower and upper end extreme matrices, $P$ and $Q$. For $A=\left\{a_{i j}\right\} \in \boldsymbol{R}^{n \times n}, P=\left\{p_{i j}\right\} \in \boldsymbol{R}^{n \times n}$ and $Q=\left\{q_{i j}\right\} \in \boldsymbol{R}^{n \times n}$, $A \in N[P, Q]$ implies that $p_{i j} \leq a_{i j} \leq q_{i j}, i, j=1, \cdots, n$. A matrix set $V[P, Q]$ where $A=\left\{a_{i j}\right\} \in V[P, Q]$ satisfies $a_{i j}=p_{i j}$ or $q_{i j}, i, j=1, \cdots, n$ is called vertex matrix 
corresponding to $N[P, Q]$. Obviously, $\mathrm{P}$ and $Q$ are included in this set. In this paper, we will exclusively consider a class of $n \times n$ matrices that have only real eigenvalues, with the notation $\mathscr{R}_{n}$. A typical example of a matrix $A \in \mathscr{R}_{n}$ is a symmetric matrix $A=A^{\prime}$, where $(')$ is a transpose symbol. Another example is a tridiagonal matrix, called a Jacobi matrix, having the form of :

$$
A=\left[\begin{array}{cccccc}
a_{11} & a_{12} & 0 & \cdot & 0 & 0 \\
a_{21} & a_{22} & a_{23} & 0 & \cdot & 0 \\
0 & a_{32} & a_{33} & a_{34} & . & 0 \\
\cdot & \cdot & \cdot & \cdot & \cdot & \cdot \\
0 & 0 & 0 & \cdot & a_{n n-1} & a_{n n}
\end{array}\right]
$$

with

$$
a_{i+1} a_{i+1 i}>0, \quad i=1, \cdots, n-1
$$

It is known that the eigenvalues of this matrix are all real ${ }^{1}$. We will further focus on a subclass of $\mathscr{R}_{n}$, denoted by $\mathscr{R}_{n}(r, k)$, by restricting the eigenvalue distribution to the situation that $r(0 \leq r \leq n)$ eigenvalues are in the interval $(k, \infty)$ while the remaining $(n-r)$ are in $(-\infty, k)$. Apparently, all the matrices in $\mathscr{R}_{n}(0,0)$ are Hurwitz stable and symmetric matrices in $\mathscr{R}_{n}(n, 0)$ are positive definite. If a matrix belonging to $\mathscr{R}_{n}(0,0)$ has no repeated eigenvalues, it is a system matrix of continuous-time aperiodic systems ${ }^{3}$. As an imaginary axis counterpart, we also consider a class of $2 n$-th order square matrices whose eigenvalues are all pure imaginary, with notation $\mathscr{I}_{2 n}$. Similarly, the subset $\mathscr{I}_{2 n}(r, k)$ of $\mathscr{I}_{2 n}$ means that every its member matrix has $2 r(0 \leq r \leq n)$ imaginary eigenvalues with modulus greater than $k$ and $2(n-r)$ ones with modulus less than $k$. A set of real numbers is said to be sign definite, if all its elements are of the same sign. For $A=A^{\prime}, A<(>) 0$ means $A$ is negative(positive) definite. Throughout the paper, symbols for matrices are used also for a set of matrices, if they apply to every member matrix. For symmetric interval matrices $S[P, Q]$, that is, $N[P, Q]$ with constraints $A$ $=A^{\prime}, P=P^{\prime}$ and $Q=Q^{\prime}$, we know that ${ }^{2), 8)}$ :

$$
\begin{aligned}
& S[P, Q]<0 \text { (Hurwitz stable) } \\
& \quad \Longleftrightarrow V[P, Q]<0 \text { (Hurwitz stable) }
\end{aligned}
$$

or

$$
S[P, Q] \subset \mathscr{R}_{n}(0, \overline{0}) \Longleftrightarrow V[P, Q] \subset \mathscr{R}_{n}(0,0) \quad(4)
$$

The main aim of this paper is to show that, so far as $N[P, Q] \subset \mathscr{R}_{n}, S[P, Q]$ and $\mathscr{R}_{n}(0,0)$ in the above relation can be replaced by $N[P, Q]$ and $\mathscr{R}_{n}(r, k)$, respectively. Note again that $S[P, Q] \subset N[P, Q] \cap$ $R_{n}$.

\section{Main Results}

The following theorem is the main result of this paper.

\section{[Theorem 1]}

Let $k$ be a real number and $r(0 \leq r \leq n)$ an interger. If $N[P, Q] \subset \mathscr{R}_{n}$, then we have :

$$
N[P, Q] \subset \mathscr{R}_{n}(r, k) \Longleftrightarrow V[P, Q] \subset \mathscr{R}_{n}(r, k)
$$

For the proof, two lemmas below are facilitated. [Lemma 1]

If the set,

$$
\{\operatorname{det}(A-k I): A \in V[P, Q]\}
$$

is sign definite for some real $k$, then

$$
\operatorname{det}(A-k I) \neq 0, \forall A \in N[P, Q]
$$

and vice versa.

Proof: We first show the case of $k=0$. The point of the proof is due to the fact that $\operatorname{det} A$ is multilinear with respect to its entries, $a_{i j}$. Let us look on $\operatorname{det} A$ as a scalar-valued matrix function. If $A \in N[P, Q]$, then its domain is a hyperbox in the matrix entry space. The range of the function is an interval on the real line and the value at both of the end points of the interval is taken on by some $A$ in $V[P, Q]$ because of the multilinearity of the function and convexity of the domain. Now, if the set in (6) is sign definite, then from the above fact the interval is obviously deviod of zero, leading to $(7)$. The converse claim can be shown by noticing the continuity of the function det $A$. If the function takes on 0 for some $A \in N[P, Q]$, then its range includes the origin. Due to the continuity, the end point values are of the opposite sign or either (or both when $A \equiv 0$ ) of them is vanishing, leading to a contradiction to the assumption the set ( 6 ) is sign definite. Thus, Lemma 1 is valid for $k=0$. The proof for case of $k \neq 0$ is straightforward, if we note that $\operatorname{det}(A-k I), V[P, Q]$ and $N[P, Q]$ in (6) and $(7)$ can also be written as $\operatorname{det} A, V[P-k I, Q$ $-k I]$ and $N[P-k I, Q-k I]$, respectively. Q.E.D.

Results worked out in somewhat similar spirit to Lemma 1 can be observed elsewhere. For example, it is reported in Ref. 9) that considerable reduction of computational cost can be achieved in obtaining the multivariable stability margin by making the most of 
the multilinearity.

\section{[Lemma 2]}

If $V[P, Q] \subset \mathscr{R}_{n}(r, k)$, then the set (6) is sign definite.

Proof: Among the eigenvalues of $A \in V[P, Q]$ $\subset \mathscr{R}_{n}(r, k)$, we label those that are larger than $k$ as $\alpha_{i}, i=1, \cdots, r$ and those less than $k$ as $\beta_{i}, i=1, \cdots, n-r$, that is, $\alpha_{i}>k, i=1, \cdots, r$ and $\beta_{i}<k, i=1, \cdots, n-r$ Then, we have :

$$
\operatorname{det}(A-k I)=\prod_{i=1}^{r}\left(\alpha_{i}-k\right) \prod_{i=1}^{n-r}\left(\beta_{i}-k\right)
$$

Evidently, the set ( 6 ) is sign definite.

We are now in position to prove Theorem 1.

Proof of Theorem 1 : Since $(\Rightarrow)$ is obvious, we prove $(\Leftarrow)$. In addition to the assumption that $V[P, Q]$ $\subset \mathscr{R}_{n}(r, k)$ we assume there exists a matrix $A_{1}$ such that $A_{1} \in N[P, Q]$ but $A_{1} \notin \mathscr{R}_{n}(r, k)$. Pick any $A_{0}$ in $V[P$, $Q]$. Then, $A_{0} \in R_{n}(r, k)$ by the assumption. In the matrix entry space, $A_{1}$ corresponds to a point in the hyperbox, while $A_{0}$ does to a vertex. We can there fore consider a continuous path connecting these two points within the hyperbox. We note here that eigenvalues of a matrix are continuous with respect to its entries. This, together with $N[P, Q] \subset \mathscr{R}_{n}$, means that along the above path we can find a point corresponding to, say, a matrix $A_{*}$ satisfying $\operatorname{det}\left(A_{*}\right.$ $-k I)=0, A_{*} \in N[P, Q]$. On the other hand, combining Lemmas 1 and 2 , we see $\operatorname{det}(A-k I)$ does not vanish for any $A$ in $N[P, Q]$. This contradiction leads to the conclusion.

Q.E. D.

As a possible application of this theorem, we can consider a modified aperiodicity property of uncertain systems. Aperiodicity property is demanded when excessive oscillatory behavior is undesirable. As mentioned before, for continuous-time state-space models aperiodicity corresponds, if multiple eigenvalues are allowed, to $\mathscr{R}_{n}(0,0)$. So, for this property, Theorem 1 directly works. We can also assert a discrete-time counterpart. A linear discrete system with a system matrix $A$ is said to be aperiodic (modified), if all the roots of its characteristic polynomial lie in the interval, $(0,1)$. With Theorem 1 , we immediately obtain the following result :

[Corollary 1]

Assume that the system matrix of an uncertain linear discrete system can be represented by $N[P, Q] \subset \mathscr{R}_{n}$.
Then, the system is aperiodic, if and only if all the matrices in $V[P, Q]$ are aperiodic.

Proof: The property is characterized by $N[P, Q]$ $\subset \mathscr{R}_{n}(0,1)$ and $N[P, Q] \subset \mathscr{R}_{n}(n, 0)$. By Theorem 1 , these can be rephrazed as $V[P, Q] \subset \mathscr{R}_{n}(0,1)$ and $V[P, Q]$ $\subset \mathscr{R}_{n}(n, 0)$, respectively. This implies that any matrix in $V[P, Q]$ is aperiodic.

Q.E.D.

Let us now turn to an imaginary axis counterpart of Theorem 1 . We can, in fact, obtain a similar result for real interval matrices belonging to $\mathscr{I}_{2 n}$. However, before showing the result, we need some modifications for the preliminaries in section 2 . When both the real and the imaginary parts of entries of a complex matrix are intervals on the real line, the matrix is called a complex interval matrix. We first note that for a $2 n \times 2 n$ real interval matrix $N[P, Q] \subset \mathscr{I}_{2 n}$, the set of $j A$, where $A \in N[P, Q]$ is a complex interval matrix included in the set $\mathscr{R}_{2 n}$. This in mind, it is easy to see that Lemma 1 remains valid if $\operatorname{det}(A-k I)$ is replaced by $\operatorname{det}(j A-k I)$ there. Notice that $\operatorname{det}(j A$ $-k I)$ is real for any $A \in N[P, Q] \subset \mathscr{I}_{2 n}$, because we have, for example,

$$
\begin{aligned}
\operatorname{det}(j A-k I)= & \prod_{i=1}^{r}\left(\alpha_{i}-k\right)\left(-\alpha_{i}-k\right) \\
& \times \prod_{i=1}^{n-r}\left(\beta_{i}-k\right)\left(-\beta_{i}-k\right)
\end{aligned}
$$

where $\alpha_{i}>k, i=1, \cdots, r, \beta_{i}<k, i=1, \cdots, n-r$. This formula also shows that a parallel result to Lemma 2 holds as well :

[Lemma 3]

If $V[P, Q] \subset \mathscr{I}_{2 n}(r, k)$, then the set,

$$
\{\operatorname{det}(j A-k I): A \in V[P, Q]\}
$$

is sign definite.

Following the same reasoning as in the proof of Theorem 1 with this Lemma and Lemma 1 where $\operatorname{det}(A-k I)$ is replaced by $\operatorname{det}(j A-k I)$, we arrive at the imaginary axis couterpart of Theorem 1 :

[Theorem 2]

Let $k$ be a positive number and $r$ be an integer with $0 \leq r \leq n$. Then, for any real interval matrices $N[P$, $Q] \subset \mathscr{I}_{2 n}$, we have an equivalence,

$$
N[P, Q] \subset \mathscr{I}_{2 n}(r, k) \Longleftrightarrow V[P, Q] \subset \mathscr{I}_{2 n}(r, k)
$$

\section{Remarks and an Example}

In this section, we give several remarks on the 
results of the previous section and provide a numerical example. In the definition of $\mathscr{R}_{n}(r, k), r$ denotes the number of eigenvalues that are larger than $k$. However, even if we alter "larger than" in that definition to "larger than or equal to", Theorem 1 is still in force. This seems to be obvious, considering the continuous dependency of matrix eigenvalues on matrix entries, but we will elaborate a formal proof to make the point precise. Let $\mathscr{R}_{n}(r, k)$ be the altered class of matrices, that is, all the $n \times n$ matrices whose eigenvalues are all real and $r$ of them are larger than or equal to $k$. We would like to show

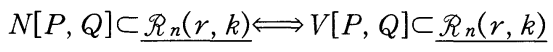

if $N[P, Q] \subset \mathscr{R}_{n}$. As before, we prove only $(\Leftarrow)$. Assume $V[P, Q] \subset \underline{\mathscr{R}_{n}(r, k)}$. Assume further $N[P, Q]$ $\not \subset \mathscr{R}_{n}(r, k)$, say, there exists $A \in N[P, Q]$ such that its $(r-1)$ eigenvalues are larger than or equal to $k$. Denote the maximum eigenvalue among the remaining $n-(r-1)$ ones by $\gamma$. Then, we can choose a small positive $\epsilon$ such that $\gamma<k-\epsilon$ and $V[P, Q] \subset \mathscr{R}_{n}(r, k$ $-\epsilon)$. We resort here to Theorem 1 where $k$ is replaced by $k-\epsilon$. Namely, we have,

$$
\begin{aligned}
& N[P, Q] \subset \mathscr{R}_{n}(r, k-\epsilon) \\
& \quad \Longleftrightarrow V[P, Q] \subset \mathscr{R}_{n}(r, k-\epsilon)
\end{aligned}
$$

Thus, for the above $A$, this relation indeed shows $A$ $\in \mathscr{R}_{n}(r, k-\epsilon)$, but $A$ has $r-1$ eigenvalues larger than $k-\epsilon$, leading to a contradiction. Other cases can be proved analogously based on the continuity argument.

Scrutinizing the proof of Corollary 1, we see that for any desired intervals, similar results to the Corollary 1 can be shown to hold, enabling us to distribute real eigenvalues of uncertain systems in accordance with prescriptions. Apparently, Theorem 1 includes the existing relation (4) as a special case. However, a merit of this specific situation is that we can further reduce the number of matrices to be checked from that of the member matrices of $V[P, Q]^{4), 6)}$.

It is believed that, as in the case of (4), some form of restrictions to $N[P, Q]$ is in general called for in order to reduce that number.

A kind of polynomial version of Theorems 1 and 2 is reported in Ref. 7), which deals with aperiodicity and periodicity of interval polynomials. Some differences actually exist, however; between interval polynomial and interval matrix cases. For one, in the results for interval polynomials, the assumption that all the roots are real is not put a priori, being included in conditions imposed to vertex polynomials. In contrast, Theorem 1 needs the assumption, $N[P, Q]$ $\subset \mathscr{R}_{n}$. The other difference is that in the polynomial case the required number of vertex polynomials to be checked is very small, irrespective of degree of polynomials. On the other hand, in the matrix case this number depends on the order of matrices anyway. We close this section with a simple numerical example of Corollary 1.

(Example)

Consider the interval matrix, $N[P, Q]$ where $P$ and $Q$ are given as

$$
P=\left[\begin{array}{ccc}
0.6 & 0.5 & 0 \\
0.2 & 0.3 & 0.2 \\
0 & 0.1 & 0.6
\end{array}\right], Q=\left[\begin{array}{ccc}
0.6 & 0.5 & 0 \\
0.2 & 0.5 & 0.3 \\
0 & 0.1 & 0.6
\end{array}\right]
$$

Since this matrix is of the form of (1) and (2), we see $N[P, Q] \subset \mathscr{R}_{3}$. The vertex matrices, $A \in V[P, Q]$ can be written as

$$
A=\left[\begin{array}{ccc}
0.6 & 0.5 & 0 \\
0.2 & a_{22} & a_{23} \\
0 & 0.1 & 0.6
\end{array}\right],
$$

where $a_{22}=0.3$ or 0.5 and $a_{23}=0.2$ or 0.3 . We can confirm that the eigenvalues of these four matrices are all located in the interval $(0,1)$ on the real line. Due to Corollary 1 , We can conclude that discrete interval systems, $\dot{z}=A z$ with $A \in N[P, Q]$ are aperiodic.

\section{Concluding Remarks}

The known result that Hurwitz stability or positive definiteness of symmetric interval matrices can be determined by checking the same property of vertex matrices is generalized. The generalization is done in two directions. One is broadening the class of matrices covered. We show that the point of above result comes from the fact that the matrices have only real eigenvalues. As the other direction of generalization, we consider the condition under which a certain number of eigenvalues are in the left of some point of the real line while the remaining ones in the right. A similar result is also shown for interval matrices having solely pure imaginary eigenvalues. The obtained theorems indicate that the so-called 
boundary implication results can be observed in not only symmetric interval matrices but also some other classes of interval matrices including symmetric ones.

\section{References}

1) S. Barnett and C. Storey : Matrix Methods in Stability Theory, Nelson, London (1970)

2) C. -L. Jiang: Sufficent Condition for the Asymptotic Stability of Interval Matrices, Internat. J. Control, 46, 1803/1810 (1987)

3) E. I. Jury : Inners and Stability of Dynamic Systems, John Wiley \& Sons, New York (1974)

4) D. Hertz:The Extreme Eigenvalues and Stability of Real Symmetric Interval Matrices, IEEE Transactions on Automatic Control, AC-37, 532/535 (1992)

5) L. V. Kharitonov: Asymptotic Stability of an Equilibrium Position of a Family of Systems of Differential Equations, Differential'nye Uravneija, 14, 2086/2088 (1978)

6) M. Mansour: Simplified Sufficient Conditions for the Asymptotic Stability of Interval Matrices, Internat. J. Control, 50, 443/444 (1989)

7) T. Mori, H. Kokame and P. Parker :Aperiodicity and Periodicity of Interval Polynomials, Proc. of 29th CDC, Honolulu, 39/40 (1990)

8) C. B. Soh: Necessary and Sufficient Conditions for Stability of Symmetric Interval Matrices, Internat. J. Control, 51, 243/248 (1990)

9) A. Sideris and R. S. S. Peña : Fast Computation of the Multivariable Stability Margin for Real Interrelated Uncertain Parameters, IEEE Transactions on Automatic Control, AC-34, 1272/1276 (1989)

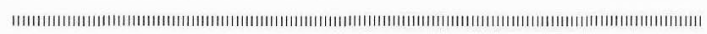

\section{Takehiro Mori (Member)}

Received the B. S. degree in 1968 and the $\mathrm{Ph}$. D. degree in 1977 all from Kyoto University in electrical engineering. In 1974, he joined research staffs of Automation Research Laboratory, Kyoto University, as a research associate. Since 1990 , he has been a professor of Kyoto Insitute of Technology. His main research interests lie in analysis of dynamical systems and stability robustness issues of control systems.

\section{Hideki Koкамe (Member)}

He received the B.E., M.E., and Dr. Eng. degrees from Kyoto University, in 1968, 1970, and 1978, respectively. From 1973 to 1980 he was working as a research associate at the same university. Since 1980 he has been with Osaka Institute of Technology, where he is presently a professor of the Department of Electrical Engineering. His research interest includes stability analysis of control systems, and computer aided control systems design.

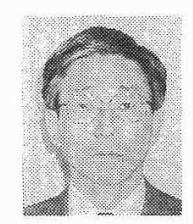

